\title{
Optical particle detection integrated in a dielectrophoretic lab-on-a-chip
}

\author{
L Cui, T Zhang and H Morgan \\ Bioelectronics Research Centre, Department of Electronics and Electrical Engineering, \\ University of Glasgow, Glasgow G12 8LT, UK
}

Received 19 March 2001, in final form 1 November 2001

Published 11 December 2001

Online at stacks.iop.org/JMM/12/7

\begin{abstract}
The design and fabrication of a dielectrophoretic 'lab-on-a-chip' device for bioparticle processing and counting is presented. The device consists of a multi-layer travelling wave dielectrophoretic electrode array for manipulating particles and/or fluids, microchannels for delivering samples and optical fibres for counting particles and/or measuring their velocities. Single particles were detected optically using either light scattering or fluorescence emission. The technology described in this study is potentially applicable to a range of particulate diagnostic systems.
\end{abstract}

(Some figures in this article are in colour only in the electronic version)

\section{Introduction}

Current trends in analytical science are directed towards miniaturization and integration, with many analytical processes carried out on a single microchip [1-3]. Such devices require integration of a number of sub-components into a single device, including sample delivery system, bio/chemical reactor and on-line detection. Optical methods are often employed for detection and these are often fabricated from integrated waveguides and/or optical fibres [4-6]. The main challenges in developing a 'lab-on-a-chip' are the integration of functional sub-units into a single practical system that can be made using realizable fabrication methods. In this work we describe the integration of a number of separate systems to produce a particle fractionation system. The device uses microelectrode arrays for particle and/or fluid movement and embedded fibreoptics for particle detection and counting.

The device is based on the use of travelling wave dielectrophoresis (twDEP) to induce particle movement and/or fractionation [7]. An array of interdigitated electrodes generates a non-uniform electric field which causes polarizable particles to move either by dielectrophoresis (DEP) and/or twDEP. Particles move under twDEP at a rate that depends on the ratio of the imaginary to real part of the effective polarizability of the particle together with their size [8]. These devices have shown potential for characterizing and sorting cells and micro-organisms [9-12] as well as for solid-state electrohydrodynamic pumping of liquid [13]. The fabrication of large-area twDEP devices has been presented previously [9] and many of the relevant fabrication technologies are available in the literature [14-18]. The twDEP devices incorporate microelectrode arrays fabricated using multilayer fabrication techniques with sealed planar microfluidic channels, including sample inlets and outlets. We have now extended this technology to enable fibreoptics to be embedded into the fluid channels for particle detection. The fibres are aligned and sealed within microfabricated grooves perpendicular to the fluid channel. A novel layout of optical windows has been implemented which enables either fluorescent or nonfluorescent particles to be detected. Using this layout, single particles can be counted and their twDEP velocities measured.

\section{Design and fabrication}

A schematic diagram outlining the operating principle of the particle detection system is shown in figure 1. This figure shows optical fibres abutting a microchannel through which particles move by twDEP. The system can be used to detect particles in one of two ways: (a) by scattering of the incident light and (b) by detecting fluorescence emission from a labelled particle. In the design shown, two pairs of optical fibres are placed and fixed in grooves in the walls of a microfluidic channel. The channel was $200 \mu \mathrm{m}$ wide and the distance between two pairs of fibres was $2 \mathrm{~mm}$. Figure 1(a) shows non-fluorescent particles (such as latex beads) moving across the optical path between the two fibres. Light from a laser diode is coupled through one fibre which is positioned directly opposite another identical fibre. When a particle passes the optical window, the incident light is scattered (or absorbed) reducing the intensity of the transmitted light. This change in 
(a)

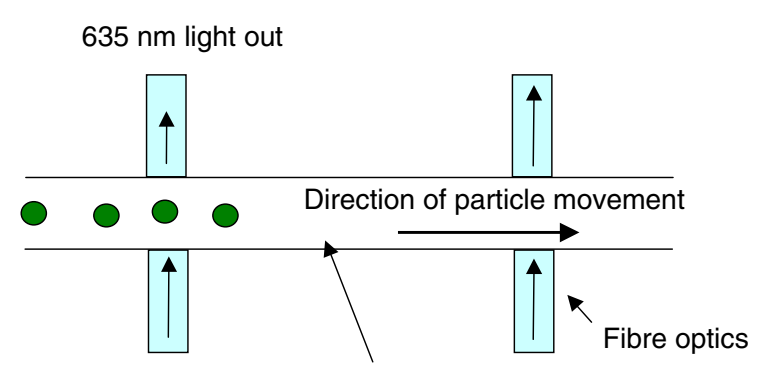

$635 \mathrm{~nm}$ light in

Micro-fluidic

channel

(b)

Detection by Scattering

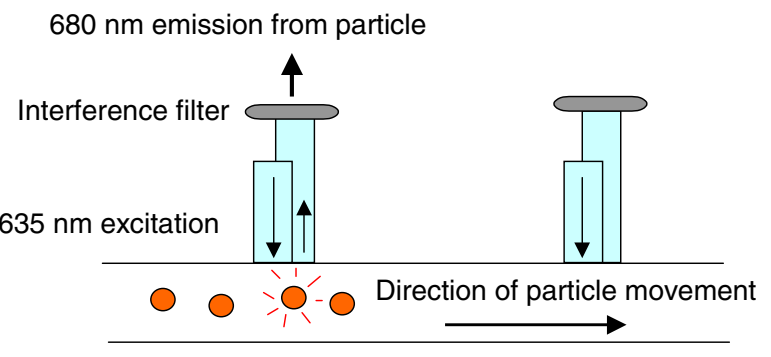

$680 \mathrm{~nm}$ emission

\section{Detection by Fluorescence}

Figure 1. A schematic diagram outlining the principle of the particle detection system. Two designs have been implemented: (a) detection by light scattering and $(b)$ fluorescence detection.

light level is measured by a photodiode and recorded as series of pulses.

The fluorescence detection method is similar and is shown in figure $1(b)$. When fluorescent particles pass across the optical window, they are excited by the light of an appropriate wavelength $(635 \mathrm{~nm}$ for the fluorescent beads used in this work). The emitted light, which is of longer wavelength (680 $\mathrm{nm}$ for the same beads), is transmitted, via a detection fibre and an interference filter $(680 \mathrm{~nm})$, to a photodiode.

Using these techniques the number of particles traversing the optical window can be determined by counting the number of signal peaks (decreasing or increasing) from the photodiode. This assumes that particles do not overlap each other while passing the window. The velocity of a single particle can be measured by using two sets of fibres arranged consecutively along a channel as shown in figure 1. If the distance is known, then by measuring the time taken for a particle to pass between each optical window, the particle velocity can be calculated.

Figure 2 shows a schematic diagram of the particle analysis chip. A suspension of particles (cells or latex spheres) is suspended in a carrier medium and introduced into the device using a syringe pump. Under optimal conditions fractionation of the particles occurs. The optical fibres for interrogating the particles are embedded in channels fabricated in the base plane of the device as shown in the figure. The device is encapsulated with a transparent lid to assist with particle observation.
Figure 3 shows a photograph of a completed device. It comprises a twDEP electrode structure, cross-shaped microfluidic channel and parallel grooves for embedding optical fibres. The fluid channels and alignment grooves were fabricated from SU-8 1070 resist (SOTEC Microsystems, Switzerland). The final layer was approximately $130 \mu \mathrm{m}$ thick and the fibre alignment grooves were 130 or $150 \mu \mathrm{m}$ wide respectively.

The SU-8 layer was processed by dispensing SU-8 onto the electrode structure, which had been baked in an oven for $30 \mathrm{~min}$ at $120^{\circ} \mathrm{C}$ for dehydration. The substrate was spun at $500 \mathrm{rpm}$ for 5 , then accelerated to $750 \mathrm{rpm}$ within $10 \mathrm{~s}$ and kept at $750 \mathrm{rpm}$ for $15 \mathrm{~s}$. It was then soft-baked on a hotplate at $90{ }^{\circ} \mathrm{C}$ for 105 min until the film was no longer sticky after cooling slowly. The SU-8 film was exposed to UV light $(436 \mathrm{~nm})$ for $200 \mathrm{~s}$ through a printed lithographic acetate mask. The mask design was created using Corel Draw ${ }^{\circledR}$ and a high-resolution transparency was produced from the design file using a high-resolution printer (Reproscan Scotland Ltd). The SU-8 film was post-exposure-baked at $90{ }^{\circ} \mathrm{C}$ for $15 \mathrm{~min}$ on a hotplate to complete the cross-linking. Development was carried out in the SU-8 developer and finally the device was thoroughly rinsed in IPA.

The device was sealed with a glass cover slide. This was bonded by spinning a very thin layer of SU-8 1040 (SOTEC Microsystems, Switzerland) onto the cover slide. The slide was spun at $500 \mathrm{rpm}$ for $5 \mathrm{~s}$, accelerated to $1000 \mathrm{rpm}$ within $5 \mathrm{~s}$, and kept at $1000 \mathrm{rpm}$ for $20 \mathrm{~s}$. It was then placed face to face with the microchannels and soft-baked on a hotplate at $90{ }^{\circ} \mathrm{C}$ for $10 \mathrm{~min}$. The SU-8 film was exposed to UV light $(436 \mathrm{~nm})$ for $20 \mathrm{~s}$ through the cover slide using another acetate mask, then post-exposure-baked at $90{ }^{\circ} \mathrm{C}$ for $8 \mathrm{~min}$ on a hotplate. Development was carried out thoroughly to make sure there were no blockages in the channels and grooves. The optical fibres (OZ Optics, Canada) were cut by an optical clipper, inserted into the grooves, aligned using a microscope and gluefixed to the planar device using fast-cure epoxy Permabond.

Figure 4(a) shows a photograph of the microfluidic channel with two optical fibres in the SU-8 grooves running perpendicular to the main channel. The mask was designed such that the width of the grooves was the same as the outer diameter of the fibre. Thus in principle a pair of fibres would be exactly aligned after placing in the grooves. In practice, there was usually a slight variation between the finished SU-8 structure and the original design so that often the groove was wider than the fibre as shown in figure $4(a)$. The final alignment of fibres was then carried out under a microscope. The fibres were permanently fixed into the grooves using epoxy adhesive. The photograph of figure 4 shows how the optical fibres are aligned using the SU-8 grooves. Figure 4(b) shows a photograph of the finished device containing a twDEP electrode, microfludic channel, fibre grooves and a pair of fixed fibres. The transmission of laser light between the pair of fibres can also be seen. In this photograph, the twDEP electrodes can clearly be seen as the alternating black and white stripes. Figure 4(c) shows a photograph of a completed device with the capillaries for sample fluid inlets and outlets, together with two pairs of fibres for particle interrogation. 


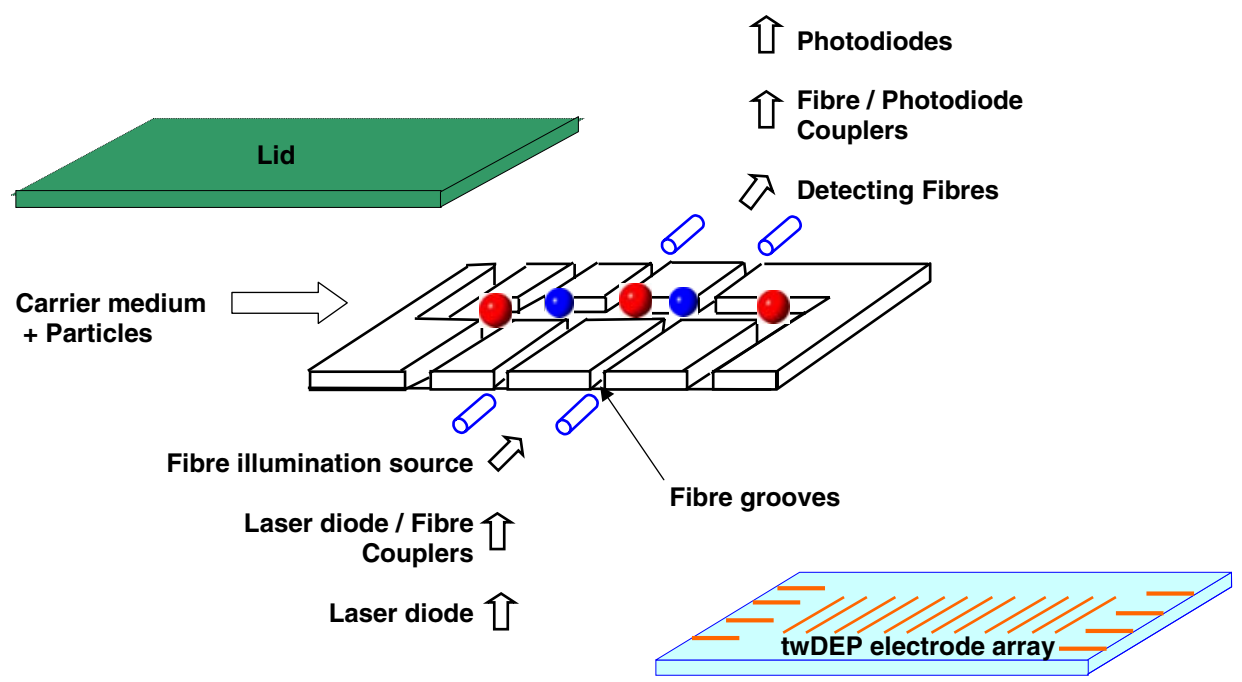

Figure 2. A schematic diagram of the integrated particle analysis chip.

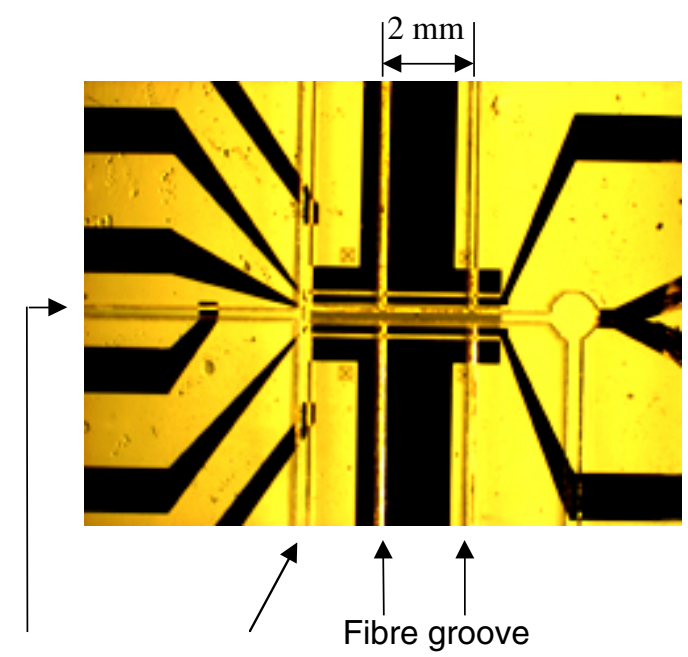

Micro-fluidic channel

Figure 3. Photograph of a completed device, comprising twDEP electrode structure, cross-shaped microfluidic channel and parallel grooves for embedding optical fibres.

\section{Experimental details}

The test particles were polystyrene microspheres with diameters of 10 and $45 \mu \mathrm{m}$ (purchased from Polysciences Europe GmbH, Germany). For fluorescent detection, $15 \mu \mathrm{m}$ diameter fluorescent polystyrene microspheres were used (Molecular Probes, USA) which absorbed at $635 \mathrm{~nm}$ and emitted in the region of $680 \mathrm{~nm}$. Prior to use, the beads were washed in de-ionized water ( $\mathrm{RO}$ water). They were then re-suspended in $\mathrm{RO}$ water, with conductivity of $0.1 \mathrm{mS} \mathrm{m}^{-1}$, which was measured using a conductivity meter (JENWAY 4071).

The twDEP electrodes beneath the microfluidic channel and fibre grooves were energized using a four-phase signal applied with a phase order of $0-90^{\circ}-180-270^{\circ}$. For all the measurements, the frequency of the signal was $0.2 \mathrm{MHz}$ and

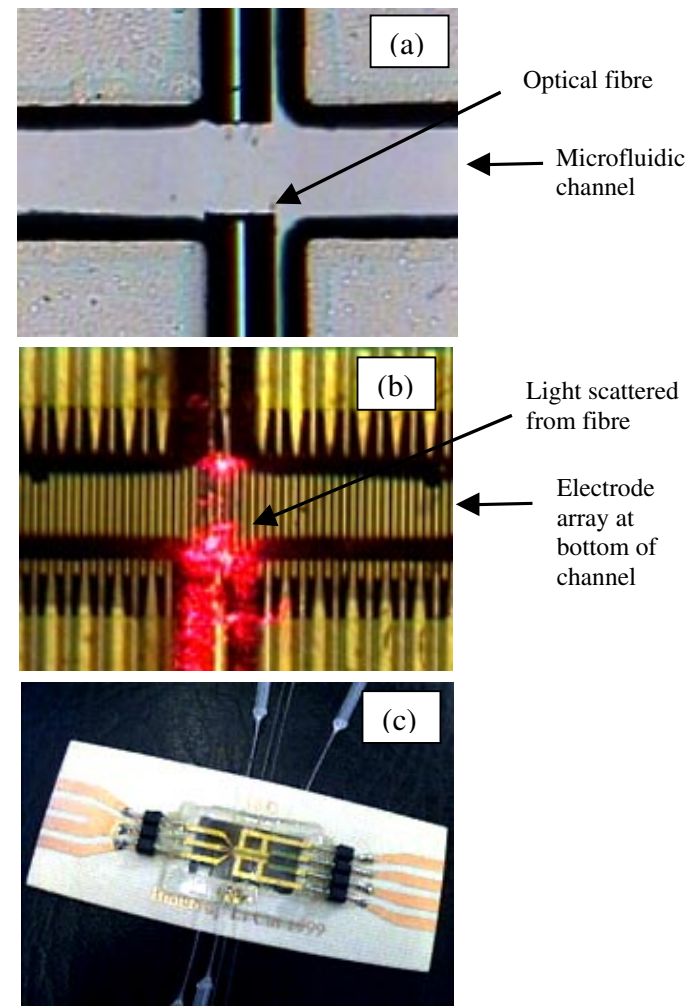

Figure 4. Photographs showing (a) the microfluidic channel with two optical fibres held in grooves perpendicular to the main channel; (b) the device containing the twDEP electrode array, microfludic channel, fibre grooves and a pair of fixed fibres. The laser light transmission between the pair of fibres can also be seen; $(c)$ a complete integrated device with the capillaries for fluid inlets and outlets and two pairs of fibres for particle interrogation.

the potential was $3 \mathrm{~V}$ (peak to peak). The twDEP electrode array had an electrode width and gap of $10 \mu \mathrm{m}$.

The optical detection consisted of two parts: the light source and the detection system. Light was coupled into the channel using a high-power fused silica multimode fibre optic 
patch cord (OZ Optics, Canada) with a $50 \mu \mathrm{m}$ diameter core, $125 \mu \mathrm{m}$ diameter cladding and a $900 \mu \mathrm{m}$ diameter jacket. Wavelength of operation was 180 to $700 \mathrm{~nm}$ with a numerical aperture of 0.22 . Before use the fibre jacket was removed so that only the core and cladding were inserted into the groove. A core diameter of $50 \mu \mathrm{m}$ was chosen to match the levitation height of the beads, which was typically in the range of 30$40 \mu \mathrm{m}$ [8]. This meant that on average the levitated particles were level with the core of the fibre, leading to better detection sensitivity.

A $10 \mathrm{~mW}, 635 \mathrm{~nm}$ laser diode (OZ Optics, Canada) was used as the excitation source. This was connected to the fibre optic patch cord using a laser diode to fibre coupler (OZ Optics, Canada). For the light scattering experiments, the receiving fibre was coupled to a photodiode with a lens style fibre to photodiode coupler (OZ Optics, Canada). For fluorescence detection, a $680 \mathrm{~nm}$ interference filter (Oriel, UK) was placed immediately before the photodiode. The bandwidth of the filter was $10 \mathrm{~nm}$ so that only light emitted by the fluoroprobe reached the photodiode. The photodiode detector signal was amplified, sampled with an analogue-to-digital converter and the data stored on a personal computer.

\section{Results}

\subsection{Detection by scattering}

At a frequency of $0.2 \mathrm{MHz}$ and a potential of $3 \mathrm{~V}$ (peak to peak), particles moved along the channel. When a single particle crossed an optical window a marked change in the intensity of the light was detected by the photodiode. This is illustrated in figure 5 which shows the $\%$ change in the signal from the photodiode plotted against time when two $45 \mu \mathrm{m}$ beads crossed one optical window. Figure 5(a) is a photograph showing the two beads in the channel. It can be seen that when the light from the emission fibre was scattered by the passing particles the signal from the photodiode detector decreased by as much as $70 \%$. (The light was not filtered in this case.) The variation in the light level between different beads was mainly due to differences in the lateral position of the particles within the channel. A larger change was measured when the particle was closer to the detection fibre end, as expected. The number of particles travelling down the channel could be determined by counting the number of peaks, providing of course that the beads are not stuck together or that two or more beads do not cross a window simultaneously. One way of overcoming this limitation is by using a low concentration of the sample or making the channel much narrower. It was not possible to distinguish beads of different size using this method, since the channel was too wide. In order to count or detect different types of particle within a sample, fluorescent labelling and detection method were used.

Beads of $10 \mu \mathrm{m}$ diameter were also analysed. However, in this case the signal was too low to be reliably detected, although light scattering was observed under the microscope. Increasing the sensitivity of the detection system or decreasing the fibre core diameter would resolve this problem. (a)

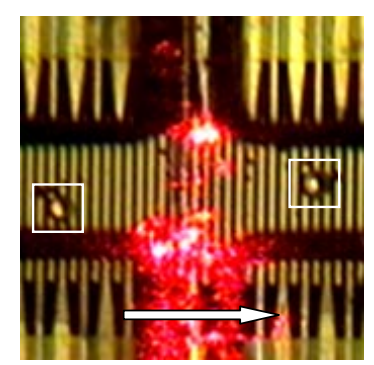

(b)

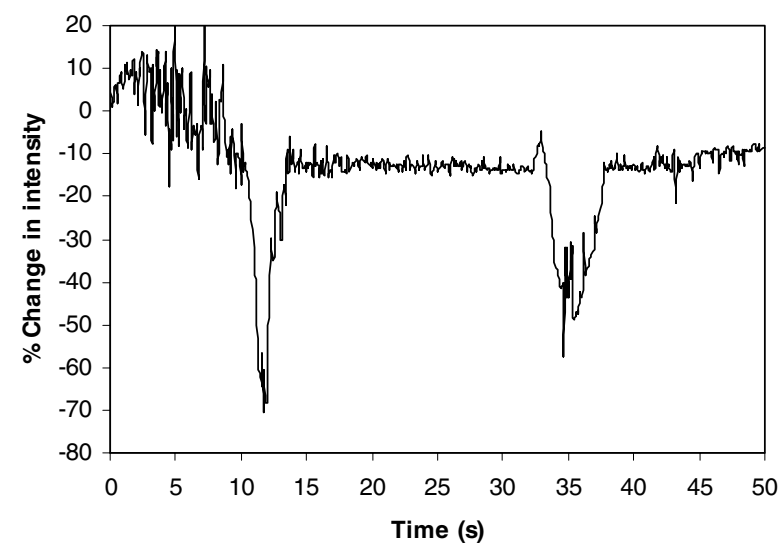

Figure 5. (a) Photograph showing two $45 \mu \mathrm{m}$ beads traversing one optical window (shown by the squares). The $\%$ change in the photodiode detector signal versus time of measurement is shown in $(b)$. The latex beads were suspended in RO water and the measurements were carried out at a frequency of $0.2 \mathrm{MHz}$ with a potential of $3 \mathrm{~V}$ (peak to peak).

\subsection{Fluorescence detection}

In order to detect the fluorescence signal from the beads, an interference filter was placed before the photodiode, allowing light in the range of $675-685 \mathrm{~nm}$ through. The fluoroprobe in the particle is excited by light of $635 \mathrm{~nm}$ wavelength and emits in the region of $680 \mathrm{~nm}$. Figure 6(a) shows photographs of a single $15 \mu \mathrm{m}$ diameter fluorescent bead approaching the excitation light source (a box is drawn around the bead). The images were taken at times of 17,28, 29 and $32 \mathrm{~s}$ respectively. As shown in figure $6(a)$, outside the region of excitation the fluorescent bead was dark. As it approached the excitation source, the bead fluoresced and appeared brighter. Figure 6(b) shows the intensity of the emitted light measured when a single $15 \mu \mathrm{m}$ diameter fluorescent bead passes the photodiode fibre.

For fluorescence detection, two fibres, one for excitation and another for detection, were positioned on top of each other in the same groove on the same side of the channel, as shown in figure $1(b)$. This arrangement reduced the background signal to nearly zero and increased the sensitivity of signal detection. Experiments showed that if the excitation and detection fibres were placed opposite each other (as for the light scattering measurement), the background signal was high (even with the interference filter) and the fluorescent signal could not be detected so that the noise obscured the florescence emission from the bead. When the source and detection fibres 
(a)

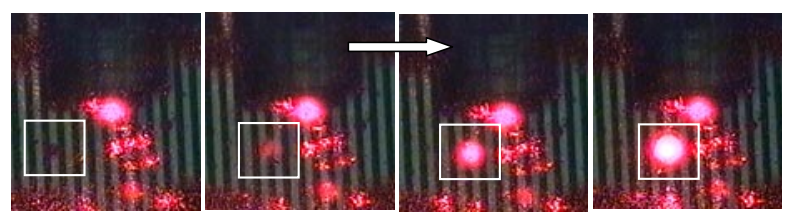

(b)

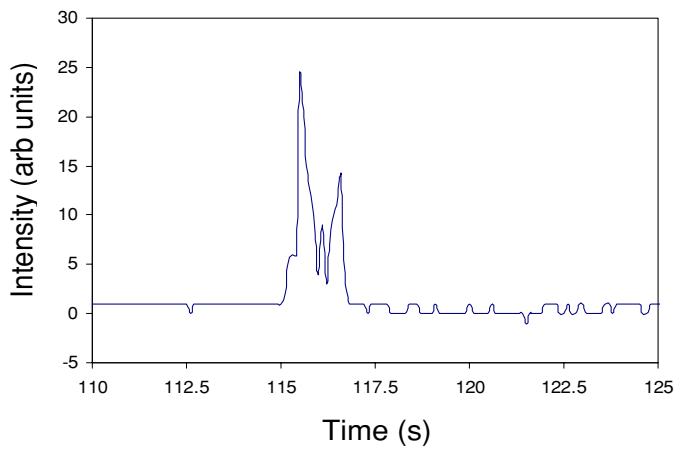

Figure 6. (a) Photographs of travelling wave induced motion of a single $15 \mu \mathrm{m}$ diameter fluorescent bead (shown by the square) as it approaches the excitation light source and emits light; and $(b)$ the intensity of the emitted fluorescence from the single $15 \mu \mathrm{m}$ fluorescent bead as detected by a photodiode.

(a)

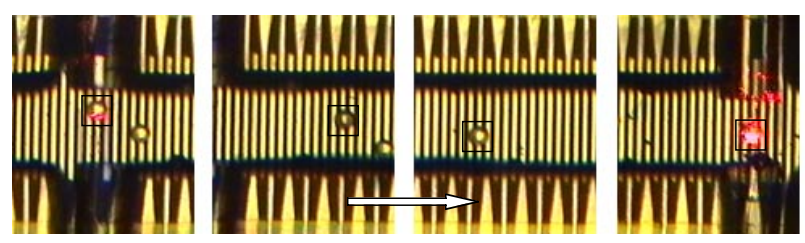

(b)

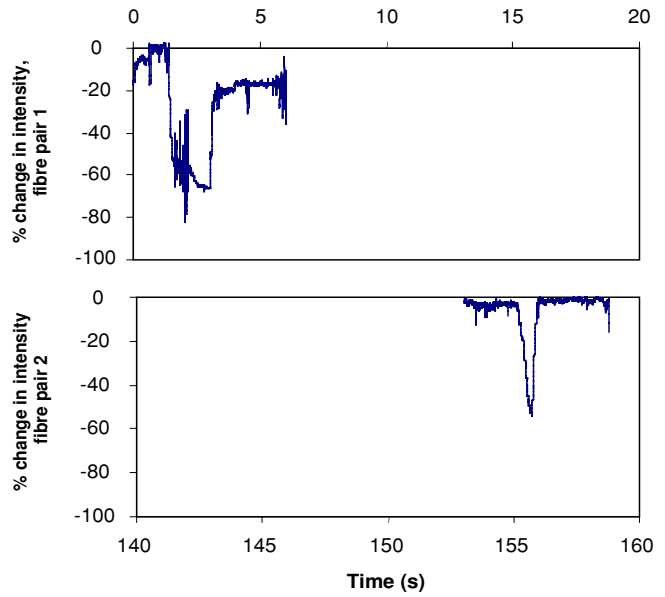

Figure 7. (a) Sequence of images showing twDEP motion of a 45 $\mu \mathrm{m}$ diameter latex bead moving down the channel at a frequency of $0.2 \mathrm{MHz}$ and an applied potential of $3 \mathrm{~V}$ (peak to peak). (b) The \% change in signal from the detector versus time for two separate pairs of fibres. The total time required to move from one optical window to the other ( $2 \mathrm{~mm}$ separation) was $153 \mathrm{~s}$. The bead velocity is therefore $13 \mu \mathrm{m} \mathrm{s}^{-1}$.

were arranged so that they pointed in the same direction, the baseline was nearly zero and sensitivity was much increased.
This experiment demonstrates the principle of fluorescence particle detection within a microchannel. The system could be developed further to enable the detection and sorting of a range of particles using different wavelength fluorescent labels.

\subsection{Particle velocity measurement}

The velocity of a particle was determined by measuring the time taken for it to travel between two pairs of fibres. Figure $7(a)$ shows a sequence of images of a $45 \mu \mathrm{m}$ diameter latex particle moving down the channel under twDEP.

The series of pictures shows the particle first of all traversing one optical window then passing a second window, $2 \mathrm{~mm}$ down-stream. Figure $7(b)$ shows the $\%$ change in the signal at the detector versus the time of measurement (in seconds) for two separate pairs of fibres. The total time required to travel the distance between two optical windows was $153 \mathrm{~s}$, the distance between two pairs of fibres was $2 \mathrm{~mm}$ so that bead velocity can be calculated as $13 \mu \mathrm{m} \mathrm{s}^{-1}$.

\section{Conclusions}

The design and fabrication of a twDEP 'lab-on-a-chip' device for bioparticle detection have been presented. An optical system has been designed capable of detecting particles moving within a twDEP device either using light scattering or fluorescence. The detection sensitivity of the system is primarily limited by the spatial positioning of the optical windows within the channel and to a lesser extent the photodiode sensitivity, which could be increased by making the channel narrower at the detection point. Such a method has been developed to count single particles where electrokinetic methods were used to control fluid movement [19]. Several different cell types could be simultaneously detected and counted by using a panel of fluorescent antibodies and exciting fluorescence using different wavelengths of light. The fabrication methods described in this paper could be used to develop miniaturized particle diagnostic systems where counting and/or sorting of cells (including fluorescently labelled) is required.

\section{Acknowledgments}

The authors wish to acknowledge EPSRC for financial support (grant no GR/M31255).

\section{References}

[1] Verpoorte S 2000 Nanoscale chemical analysis Trends Anal. Chemi. 19 350-1

[2] Kricka L J 1998 Miniaturization of analytical systems Clinical Chem. 44 2008-14

[3] Burns M A et al 1998 An integrated nanoliter DNA analysis device Science 282 484-87

[4] Robitaille L, Callendar C L and Noad J P 1996 Design and fabrication of low-loss polymer waveguide components for on-chip optical interconnection IEEE Photonics Tech. Lett. 8 1647-9

[5] Leistiko O and Jensen P F 1998 Integrated bio/chemical microsystems employing optical detection: the clip-on J. Micromech. Microeng. 8 148-50 
[6] Eldada L and Shacklette L W 2000 Advances in polymer integrated optics IEEE J. Selected Topics in Quantum Electron. 6 54-68

[7] Pethig R 1996 Dielectrophoresis: using inhomogeneous A.C. electric fields to separate and manipulate cells Crit. Rev. Biotechnol. 16 331-48

[8] Cui L, Holmes D and Morgan H 2001 The dielectrophoretic levitation and separation of latex beads in microchips Electrophoresis 22 3893-901

[9] Cui L and Morgan H 2000 Design and fabrication of travelling wave dielectrophoresis structures $J$. Micromech. Microeng. $1072-9$

[10] Chan K L, Green N G, Hughes M P and Morgan H 1998 Cellular characterisation and separation: dielectrophoretically activated cell sorting (DACS) Proc IEEE Conf. Eng. Medi. and Biol. (Hong kong) pp 2953-6

[11] Morgan H, Green N G, Hughes M P, Monaghan W and Tan T C 1997 Large-area travelling-wave dielectrophoresis particle separator J. Micromech. Microeng. 7 65-70

[12] Talary M S, Burt J P H, Tame J A and Pethig R 1996 Electromanipulation and separation of cells using travelling electric fields J. Phys. D: Appl. Phys. 29 2198-203

[13] Fuhr G, Hagedorn R, Müller T, Benecke W and Wagner B 1992 Microfabricated electrohydrodynamic (EHD) pumps for liquids of higher conductivity J. Microelectromech. Syst $1141-6$

[14] Shaw J M, Gelorme J D, LaBianca N C, Conley W E and Holmes S J 1997 Negative photoresists for optical lithography IBM J. Res. Dev. 41 0018-8646

[15] Lorenz H, Despont M, Fahrni N, LaBianca N, Renaud P and Vettiger P 1997 SU-8: a low-cost negative resist for MEMS J. Micromech. Microeng. 7 121-4

[16] Lee K Y, LaBianca N, Rishton S A, Zolgharnain S, Gelorme J D, Shaw J and Chang T 1995 Micromachining application of a high resolution ultrathick photoresist $J$. Vac. Sci. Technol. B 13 3012-16

[17] Jackman R J, Floyd T M, Schmidt M A and Jensen K F 2000 Development of methods for on-line chemical detection with liquid-phase microchemical reactors using conventional and unconventional techniques Proc. $\mu T A S$ 2000 Symp. Micro Total Analysis Systems 2000 pp 155-8

[18] Zhang J, Tan K L, Hong G D, Yang L J and Gong H Q 2001 Polymerization optimization of SU-8 photoresist and its applications in microfluidic systems and MEMS J. Micromech. Microeng. 11 20-6

[19] Fu A Y, Spence C, Scherer A, Arnold F H and Quake S R 1999 A microfabricated fluorescence-activated cell sorter Nature Biotech. 17 1109-11 